\title{
Predicting Solar Cycle 24 Using a Geomagnetic Precursor Pair
}

\author{
W. Dean Pesnell
}

Received: 30 May 2013 / Accepted: 31 December 2013 / Published online: 22 January 2014

(C) The Author(s) 2014. This article is published with open access at Springerlink.com

\begin{abstract}
We describe using Ap and $\mathrm{F}_{10.7}$ as a geomagnetic-precursor pair to predict the amplitude of Solar Cycle 24. The precursor is created by using $\mathrm{F}_{10.7}$ to remove the direct solar-activity component of Ap. Four peaks are seen in the precursor function during the decline of Solar Cycle 23. A recurrence index that is generated by a local correlation of Ap is then used to determine which peak is the correct precursor. The earliest peak is the most prominent but coincides with high levels of non-recurrent solar activity associated with the intense solar activity of October and November 2003. The second and third peaks coincide with some recurrent activity on the Sun and show that a weak cycle precursor closely following a period of strong solar activity may be difficult to resolve. A fourth peak, which appears in early 2008 and has recurrent activity similar to precursors of earlier solar cycles, appears to be the "true" precursor peak for Solar Cycle 24 and predicts the smallest amplitude for Solar Cycle 24. To determine the timing of peak activity it is noted that the average time between the precursor peak and the following maximum is $\approx 6.4$ years. Hence, Solar Cycle 24 would peak during 2014. Several effects contribute to the smaller prediction when compared with other geomagnetic-precursor predictions. During Solar Cycle 23 the correlation between sunspot number and $F_{10.7}$ shows that $F_{10.7}$ is higher than the equivalent sunspot number over most of the cycle, implying that the sunspot number underestimates the solar-activity component described by $\mathrm{F}_{10.7}$. During 2003 the correlation between aa and Ap shows that aa is $10 \%$ higher than the value predicted from Ap, leading to an overestimate of the aa precursor for that year. However, the most important difference is the lack of recurrent activity in the first three peaks and the presence of significant recurrent activity in the fourth. While the prediction is for an amplitude of Solar Cycle 24 of $65 \pm 20$ in smoothed sunspot number, a below-average amplitude for Solar Cycle 24, with maximum at 2014.5 \pm 0.5 , we conclude that Solar Cycle 24 will be no stronger than average and could be much weaker than average.
\end{abstract}

Keywords Solar cycle, predictions

W.D. Pesnell ( $\square)$

NASA Goddard Space Flight Center, Greenbelt, MD, USA

e-mail: william.d.pesnell@nasa.gov 


\section{Introduction}

Predicting solar activity is an active area of research. Our knowledge of the Sun and solar activity will be validated by testing such predictions, from the seconds that anticipate a flare to the decades needed for planning satellite missions. Numerous authors have advocated using geomagnetic precursors to predict the level of activity in an upcoming solar cycle. The value of a heavily smoothed geomagnetic index, such as aa or Kp, at solar minimum correlates with the strength of the next cycle (Ohl, 1966). To create predictions before solar minimum, geomagnetic-precursor pairs are created by removing the direct solar-activity component from the geomagnetic index, usually by a correlation fit with a solar-activity index. By removing the solar-activity component, the predictive power of the precursor is moved to an earlier phase of the solar cycle. Sargent (1978) and Ohl and Ohl (1979) developed geomagnetic-precursor pairs by removing the geomagnetic activity directly correlated with solar activity from the geomagnetic activity index to produce predictions of Solar Cycle 21. Feynman (1982) suggested that the geomagnetic-activity index aa could be separated into one component proportional to the solar-activity cycle and an "interplanetary" component that resembles the sunspot cycle, but is delayed by several years after solar maximum. The interplanetary component often peaks just before solar minimum and was shown to be a better indicator for the amplitude of the following cycle by Hathaway, Wilson, and Reichman (1999). However, Brown (1992) showed that removing the solar-activity component did not produce better predictions of Solar Cycle 22.

The frequency of solar flares and coronal mass ejections (CMEs), the main drivers of the solar-cycle component of geomagnetic activity, rises and falls with $R_{\mathrm{Z}}$ or $\mathrm{F}_{10.7}$ (Feynman, 1982); updated through Solar Cycle 23 by Pesnell (2008a). Geomagnetic activity is also produced by the high-speed solar-wind streams flowing out of the coronal holes that develop out of phase with the sunspot cycle (Luhmann et al., 2002). During the decline to sunspot minimum, the low-latitude extensions of these coronal holes produce long-lasting high-speed streams that drive recurrent geomagnetic activity. The magnetic fields that give rise to these coronal holes may be the actual precursor of the upcoming sunspot cycle, although the causal relationship is not completely understood (Wang and Sheeley, 2009).

Geomagnetic precursors were an important part of the consensus prediction for Solar Cycle 23 (Joselyn et al., 1997). However, all of the predictions for Solar Cycle 23 from geomagnetic precursor methods as evaluated by Hathaway, Wilson, and Reichman (1999) indicated a larger amplitude than was observed; although the actual amplitude was within or just outside their $2 \sigma$ error estimates. The Solar Cycle 24 Prediction Panel also relied on geomagnetic precursors as an important component of their consensus prediction (Biesecker and the Solar Cycle 24 Prediction Panel, 2007).

Predictions of the amplitude of Solar Cycle 24 using geomagnetic precursors cover a wide range of values (Pesnell, 2008b, 2012). One geomagnetic-precursor pair prediction combines aa and $R_{\mathrm{Z}}$ to predict a large amplitude for Solar Cycle 24 of $160 \pm 25$ for the maximum of the smoothed sunspot number (Hathaway and Wilson, 2006), although that value was later revised to below-average. A geomagnetic precursor using a time-shifted Kp predicts an amplitude of 96 (Kryachko and Nusinov, 2008). The trend of solar activity predicted by the geomagnetic-precursor pair of Hathaway and Wilson (2006) disagrees by a large amount with the predictions based on the solar polar field (Schatten, 2005; Svalgaard, Cliver, and Kamide, 2005). Reducing or understanding these discrepancies is necessary to give confidence to our ability to predict the amplitude of an upcoming solar cycle.

Several issues could be present in the data used to construct a geomagnetic precursor of solar activity that would lead to an incorrect prediction. One is the adequacy of a 
geomagnetic-activity index in representing that part of geomagnetic activity that could be a precursor. Another is how accurately the sunspot number $\left[R_{\mathrm{Z}}\right]$ represents solar activity. Solar Cycles 22 and 23 both had secondary peaks in $\mathrm{F}_{10.7}$ and a number of active regions in the southern hemisphere that were not as well represented in $R_{\mathrm{Z}}$. We will show that the residual in a correlation fit between $R_{\mathrm{Z}}$ and $\mathrm{F}_{10.7}$ shows that $R_{\mathrm{Z}}$ has been trending below $\mathrm{F}_{10.7}$ since about 1996 . Finally, we consider the effect of varying the width of the averaging filter and whether a weak-cycle precursor would be resolved when the filter width also includes a period of extremely high solar activity.

In this article we examine how Ap and $\mathrm{F}_{10.7}$ can be combined into a geomagneticprecursor pair to forecast the level of activity of Solar Cycle 24. The predicted amplitude is of an averaged sunspot cycle, such as described by Hathaway, Wilson, and Reichman (1994), which can be described by a single number. Details such as Gnevishev's gap, where two distinct peaks are observed, cannot be anticipated with this technique. The sensitivity of the predicted amplitude to the width of the averaging filter and association with recurrent activity is explored. Next we show that the precursor should be evaluated at the time of peak recurrent activity near solar minimum. If this condition is not satisfied, the predicted amplitude will always be overestimated. The data series are described in the Appendix.

\section{Ap and $F_{10.7}$ as a Geomagnetic Precursor Pair}

Although the aa $-R_{\mathrm{Z}}$ precursor pair has been used by several authors to predict solar activity, we have six reasons to use Ap- $\mathrm{F}_{10.7}$.

\subsection{Changes in $R_{\mathrm{Z}}$ Calibration}

One reason is the long-term reliability of $R_{\mathrm{Z}}$. Svalgaard (2012) claims that $R_{\mathrm{Z}}$ was too small in the nineteenth century. If a long-term index has a bias toward being too large in the current epoch, then predictions using that index will show a similar bias. By using the well-calibrated $\mathrm{F}_{10.7}$ as the solar-activity index we reduce these biases.

\subsection{Relative Behavior of $\mathrm{F}_{10.7}$ and $R_{\mathrm{Z}}$}

Another reason is the change in the relative behavior of $\mathrm{F}_{10.7}$ and $R_{\mathrm{Z}}$ in Solar Cycle 23 . If we assume an error of five in both $\mathrm{F}_{10.7}$ and $R_{\mathrm{Z}}$, there is a fairly linear correlation relationship between these two indices, given by $R_{\mathrm{Z}}=-63.8+1.07 \mathrm{~F}_{10.7}$. The residuals in this correlation fit $\left[\Delta R_{\mathrm{Z}}\right]$ contain two major contributions (shown by the blue line in Figure 1). One is a trend for $\Delta R_{\mathrm{Z}}$ to decrease from positive to negative during the period of time that $F_{10.7}$ is available. The other is an oscillatory component that was associated with other solar-activity observations until Solar Cycle 23. This residual shows that $R_{\mathrm{Z}}$ tends to be underestimated by $\mathrm{F}_{10.7}$ in the ascending phase of the solar cycle and overestimated in the descending phase of the solar cycle. But $\Delta R_{\mathrm{Z}}$ has remained negative ( $R_{\mathrm{Z}}$ is smaller than predicted by the correlation fit with $\mathrm{F}_{10.7}$ ) since the solar minimum in 1996 . If $R_{\mathrm{Z}}$ is too small in the current epoch, the solar-activity component of geomagnetic activity would be incompletely subtracted from aa, changing the magnitude of a precursor peak in that phase of the solar cycle. A higher value of the solar component in a precursor pair will tend to reduce the predicted level of solar activity.

We have three reasons to interpret this trend as a decrease in $R_{\mathrm{Z}}$ rather than an increase in $\mathrm{F}_{10.7}$. Svalgaard (2009) investigated how $\mathrm{F}_{10.7}$ tracks other radio-irradiance measurements, 
Figure 1 The residual of the fit between $R_{\mathrm{Z}}$ and $\mathrm{F}_{10.7}$ (straight annual averages.) The residual is shown as the blue solid line and the North-South asymmetry in the number of active regions is shown as a solid red line. The tendency of $R_{\mathrm{Z}}$ to be smaller than $\mathrm{F}_{10.7}$ appears to be increasing with time.

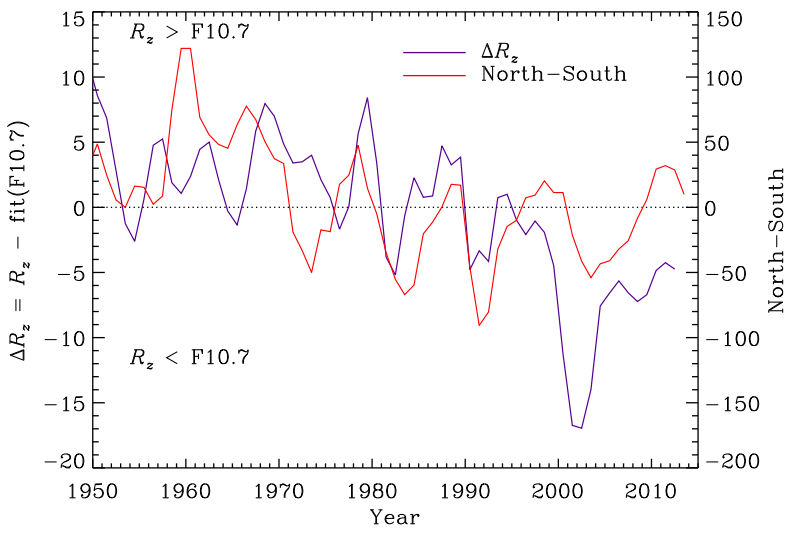

primarily from the Japanese radio observatories. He concluded that the calibration of $F_{10.7}$ can be traced through the entire time series and is not drifting to higher values in the current epoch. Tapping and Valdés (2011) showed that several solar-activity indices, especially $\mathrm{F}_{10.7}$ and $R_{\mathrm{Z}}$, are well correlated at some times and less so at others. They associated these differences with the evolution of solar activity in the different layers of the solar atmosphere. Finally, Livingston, Penn, and Svalgaard (2012) also argued that the relationship between $\mathrm{F}_{10.7}$ and $R_{\mathrm{Z}}$ appears to be drifting.

A possible explanation for some of the variation is that $\mathrm{F}_{10.7}$ tends to track the number of active regions in the Sun's southern hemisphere during the second half of Solar Cycles 22 and 23 while $R_{\mathrm{Z}}$ does not. This is illustrated by the red curve in Figure 1, where the difference in the number of active regions in both hemispheres of the Sun (the N-S asymmetry), derived from the active regions reported for each year since 1950 by the National Geophysical Data Center (NGDC) website, is plotted. The number of active regions in both hemispheres of the Sun were derived from the active regions reported for each year since 1965 on the NGDC website. Before 1965, the data values were obtained from the NGDC as yearly files containing the measured active-region locations and areas measured by the Greenwich Observatory. The overlap years were averaged.

The residual $\Delta R_{\mathrm{Z}}$ tracks the $\mathrm{N}-\mathrm{S}$ asymmetry until 1996, after which it becomes increasingly negative. The residual had not returned to the \pm 5 band at the end of 2008 . This behavior is unlike the previous three cycles, where $\Delta R_{\mathrm{Z}}$ tended to track the $\mathrm{N}-\mathrm{S}$ asymmetry.

A similar asymmetry in the location of flares, described as the relative difference of the northern and southern hemispheres, is described by Temmer et al. (2001). The asymmetry described here does have a definite variation with solar activity in the previous three cycles; early in the cycle there is an excess of northern active regions and later there is an excess of active regions in the southern hemisphere. A similar pattern is seen in the N-S asymmetry for the measured spot numbers since 1874 (Li, Gao, and Zhan, 2009). However, the southern hemisphere led the northern before Solar Cycle 19, while the North has led the South since Solar Cycle 20. Although the phasing is not perfect, fluctuations in the magneticfield strength in sunspots shows a similar solar-cycle variation (Pevtsov et al., 2013; McIntosh et al., 2013).

\subsection{Relative Behavior of aa and Ap}

Next, aa is an antipodal index, where several effects are canceled by combining stations on diametrically opposite sides of the Earth. Over time the station pairs have changed and 
offsets in aa have been suggested to maintain an accurate long-term index (Svalgaard, Cliver, and Le Sager, 2004). Ap is an average over many stations and is much less sensitive to changes in their locations. The residual in a correlation fit between aa and Ap shows a tendency for aa to be greater than the correlation fit during the decline of Solar Cycle 23, but the magnitude of that bias is very sensitive to whether or not the suggested offsets for aa are used. A lower value of the geomagnetic component in a precursor pair will tend to reduce the predicted level of solar activity. This effect is weaker than the $\Delta R_{\mathrm{Z}}$ trend discussed above.

\subsection{Time Coverage}

A third effect is the importance of the temporal coverage, or number of previous cycles, of the precursor pair. Hathaway and Wilson (2006) show a precursor from about 1870 using aa and $R_{\mathrm{Z}}$. However, their precursor has a more structured temporal dependence before 1930 than after (there are 12 maxima in the precursor during the seven solar cycles between 1870 - 1930; nine maxima in the eight solar cycles between 1930-2000). Another observation is that all of the cycles before Cycle 17 have multiple peaks in the precursor and only one has a multiple peak after Cycle $17(\approx 1960$, between Cycles 19 and 20$)$. This could be a statistical fluctuation but it could also represent a true systematic effect in the data. A different precursor pair can be examined to see if these effects are important. Because both Ap and $F_{10.7}$ are available since 1950, this pair can be examined over most of the modern period of the aa $-R_{\mathrm{Z}}$ precursor function. Using $\mathrm{F}_{10.7}$ as the activity indicator limits the number of previous cycles to five but still gives a correlation that spans a range sufficient to predict the amplitude of Solar Cycle 24.

\subsection{Effects of Smoothing Filter}

A fourth reason is that the wide smoothing filter advocated by Hathaway and Wilson (2006) encompasses both peaks of $\mathrm{F}_{10.7}$ during solar maximum in Solar Cycles 22 and 23. The presence and size of precursor peaks should be examined under other filter widths to see what effect this has on the predicted amplitude of Cycle 24. A precursor of a weak cycle will be quite small and could be disguised by the smoothing process. This was motivated by the Ap figure on the NOAA/SEC Solar Cycle Progression website (www.sec.noaa.gov/SolarCycle/), which shows peaks in 2005, 2006, and 2008 that may be more appropriate geomagnetic precursors for Solar Cycle 24.

\subsection{Effect of Magnetosphere-Solar Wind Coupling}

Finally, what is the effect of the seasonal changes in the coupling between the terrestrial magnetosphere and the solar-wind fluctuations that drive geomagnetic activity? The extraordinary solar activity in late 2003 coincided with a maximum in the semi-annual RussellMcPherron effect (Russell and McPherron, 1973; Crooker, Cliver, and Tsurutani, 1992), when the tilt of the Earth's rotation axis allows the most effective coupling between the magnetosphere and the solar wind. Without a clear understanding of this effect, it should at least be included in the uncertainty of the predicted amplitude.

\section{Construction of Ap-F $\mathbf{F}_{10.7}$ Geomagnetic Precursor Pair}

We adapted the methods originally described by Feynman (1982) and modified by Hathaway and Wilson (2006), but with Ap and $\mathrm{F}_{10.7}$ as the data streams. The construction of a 


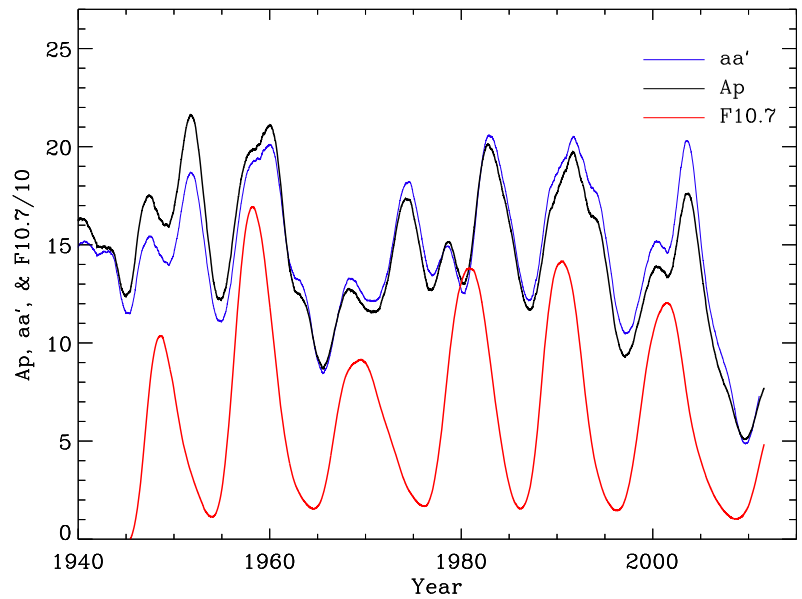

Figure 2 The averaged Ap and scaled aa indices from 1930 through 2008. The aa index was scaled by the correlation fit $\mathrm{Ap}=-4.00+0.807$ aa and is listed as aa' in the legend. A wide $(\mathrm{FWHM}=730$ days $)$ Gaussian filter was used to average the data. Note that aa' and Ap track each other until about 1980. This is particularly important for the geomagnetic-precursor analysis. Values of scaled aa are consistently higher than Ap since 1980, with the peak levels remaining constant while the peak values of Ap show a decrease over the same interval. The run of $\mathrm{F}_{10.7}$ (divided by ten) from 1950 through 2008 is shown for orientation.

Figure 3 Averaged Ap and $\mathrm{F}_{10.7}$ indices. The straight line is described by

$\operatorname{Ap}_{S A}=3.4+6.0 \times 10^{-2} \mathrm{~F}_{10.7}$. The fit represents the solar-activity contribution of Ap. After subtracting this fit from Ap, we obtain the geomagnetic precursor.

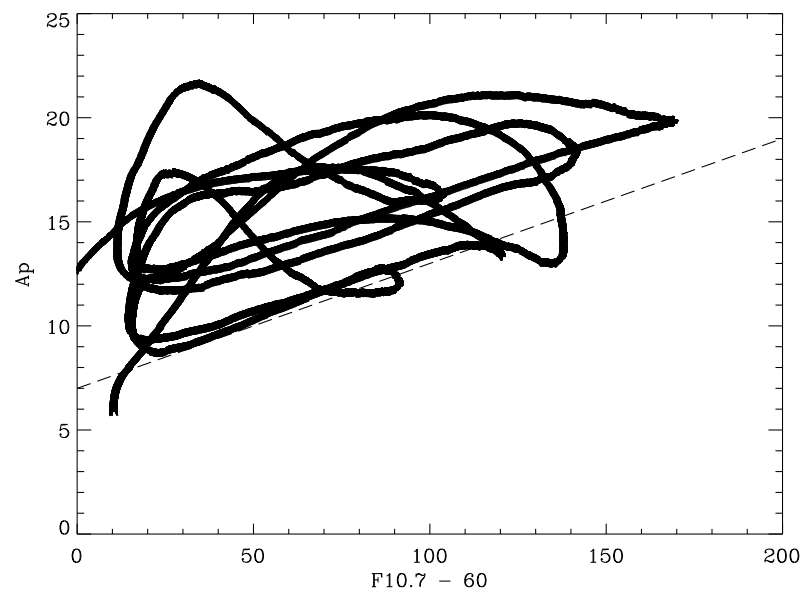

geomagnetic-precursor pair using Ap and $\mathrm{F}_{10.7}$ as the predictor/predicted pair is illustrated in Figures 2 through 5.

The data were smoothed by the Gaussian filter recommended by Hathaway and Wilson (2006) with a width parameter $\left[\sigma_{\mathrm{WG}}\right]$ that was varied from 81 to 310 days. The widest filter had a FWHM of 730 days, encompassing almost four years of data when the filter was truncated to span $\pm 2 \sigma_{\mathrm{WG}}$. The filter was truncated at \pm 620 days and divided by the normalization of the truncated filter. The truncation at $\pm 2 \sigma_{\mathrm{WG}}$ means that $4.5 \%$ of the filter was removed. Changing the width to $\pm 3 \sigma_{\mathrm{WG}}$ did not change any of the conclusions discussed here, but removing data at the recent end of the time series restricts how close to the present the precursor can be evaluated. 


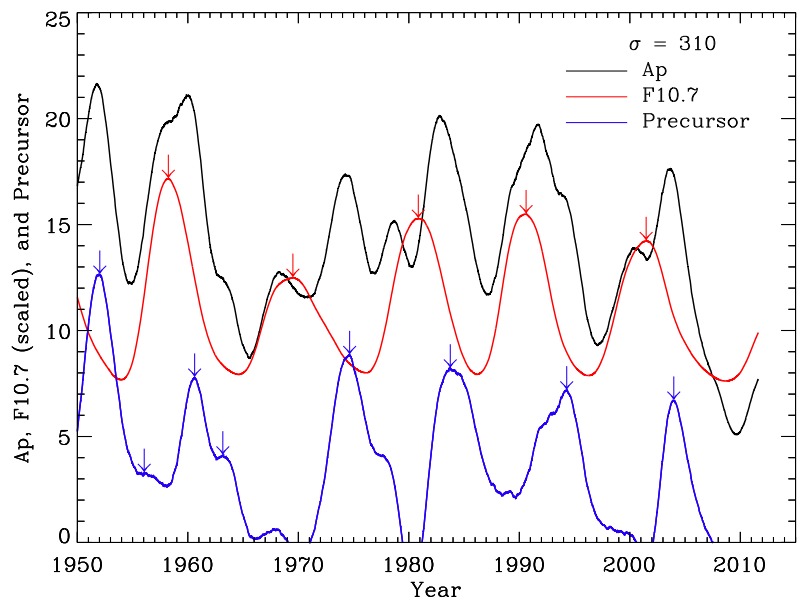

Figure 4 Temporal dependence of the interplanetary contribution to geomagnetic activity (in blue) that was found by subtracting the solar-activity component from Ap, which is then identified as the precursor. Excursions below zero were ignored. Maxima in both $\mathrm{F}_{10.7}$ and the precursor after 1950 are indicated. Values of these maxima were interpolated and used to construct the prediction points in Figure 5. The unaltered run of Ap is shown as a black line and the solar-activity component of Ap (from Equation (1)) is shown as a red line labeled as $\mathrm{F}_{10.7}$ in the legend.

Figure 5 Predictor correlation fit between Ap and $\mathrm{F}_{10.7}$. The points marked by "+"s were interpolated from Figure 4. Cycle numbers are written near the plotted symbols. The straight line shows the linear-prediction relationship, whose fit coefficients are listed in Equation (2). For $A p_{p}=0.1$ we obtain a predicted $\mathrm{F}_{10.7}$ amplitude of 122. Converting to annual-averaged sunspot number, this gives $R_{\mathrm{Z}, \mathrm{AA}}=65$, well below an average cycle of $115 \pm 40$.

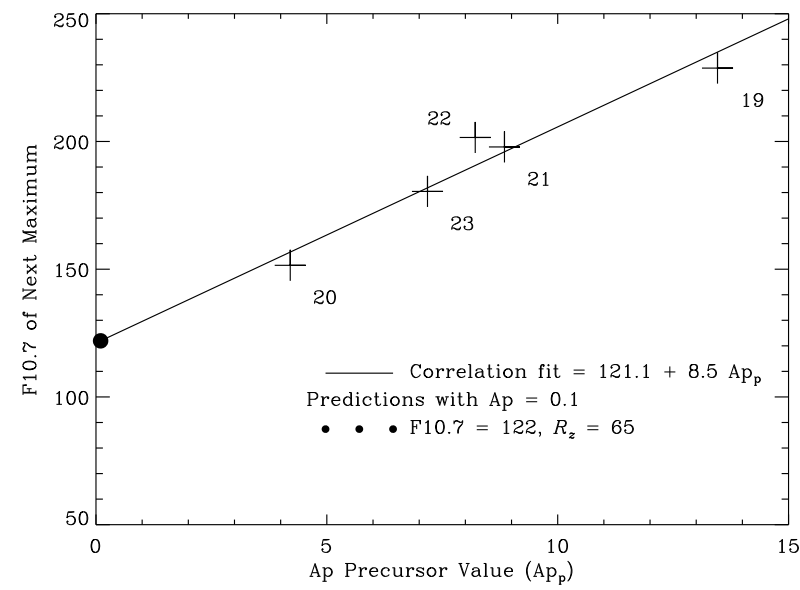

The wide-Gaussian averaged data for $\sigma_{\mathrm{WG}}=310$ days are shown in Figure 2. A fit to the lower envelope of a plot of Ap vs. $\mathrm{F}_{10.7}$,

$$
\mathrm{Ap}_{\mathrm{SA}}=3.4+6.0 \times 10^{-2} \mathrm{~F}_{10.7}
$$

is shown in Figure 3. We identified this fit as the solar-activity contribution to Ap and subtracted its value from each Ap value, leaving the interplanetary contribution, which is shown as the solid blue line in Figure 4. Maxima of $\mathrm{F}_{10.7}$ and the precursor, indicated by downward arrows in Figure 4, are read from the graph and plotted in a correlation diagram in Figure 5. Solar cycle numbers are written near the plotted symbols corresponding to that cycle pair.

The temporal dependence of the precursor in Figure 4 is similar to that of Figure 2 in Hathaway and Wilson (2006). One difference is that this peak value of the precursor de- 
Table 1 Predictions of Solar Cycle 24: all have estimated errors of \pm 20 .

\begin{tabular}{|c|c|c|c|}
\hline Precursor $\left[A p_{p}\right]$ & $\mathrm{F}_{10.7, \mathrm{AA}}$ & $R_{24, \mathrm{AA}}$ & Method \\
\hline 6.7 & 183 & 130 & $\begin{array}{l}\sigma_{\mathrm{WG}}=310 \text {-day precursor evaluated at peak } \\
\text { in } 2003\end{array}$ \\
\hline 4.8 & 165 & 115 & $\begin{array}{l}\sigma_{\mathrm{WG}}=150 \text {-day precursor evaluated at peak } \\
\text { in } 2005\end{array}$ \\
\hline 4.0 & 158 & 105 & $\begin{array}{l}\sigma_{\mathrm{WG}}=310 \text {-day precursor evaluated at time } \\
\text { of maximum in } 2005 \text { from the } \\
\sigma_{\mathrm{WG}}=150 \text { day curve }\end{array}$ \\
\hline 1.0 & 130 & 75 & $\begin{array}{l}\sigma_{\mathrm{WG}}=150 \text {-day precursor evaluated at peak } \\
\text { in } 2006\end{array}$ \\
\hline 0.1 & 122 & 65 & $\begin{array}{l}\sigma_{\mathrm{WG}}=150 \text {-day precursor evaluated at peak } \\
\text { in } 2008 \text { from the } \sigma_{\mathrm{WG}}=81 \text {-day curve }\end{array}$ \\
\hline Average & 125 & 70 & Includes the last two predictions \\
\hline
\end{tabular}

creases in a roughly linear fashion during the last three solar minima, while the aa- $R_{\mathrm{Z}}$ pair does not. The height of the second peak in the double peak around 1963 in the aa- $R_{\mathrm{Z}}$ pair has been reduced in the Ap- $\mathrm{F}_{10.7}$ pair. This could represent the asymmetry in $R_{\mathrm{Z}}$ where $\mathrm{F}_{10.7}$ does not show the same slope in the second half of the solar cycle as $R_{Z}$ (see the Appendix and Figure 1).

The straight line in Figure 5 shows the correlation relationship when the precursor for Cycle 20 is 4.2 (the second, smaller peak). A linear fit to the points in Figure 5, allowing for errors in both coordinates, is

$$
\mathrm{F}_{10.7, n+1}=120( \pm 9)+8.5( \pm 1) \mathrm{Ap}_{\mathrm{p}} \chi^{2}=4.5 .
$$

Figure 4 shows that the precursor value in 2003 for Solar Cycle 24 with $\sigma_{\mathrm{WG}}=310$ days is $\mathrm{Ap}_{\mathrm{p}}=6.7$. Using the linear fit between the annual-averaged and wide-Gaussian $\mathrm{F}_{10.7}$, $\mathrm{F}_{10.7, \mathrm{AA}}=\left(\mathrm{F}_{10.7, \mathrm{WG}}-13.3\right) / 0.898$, followed by the conversion between annual-averaged $\mathrm{F}_{10.7}$ and $R_{\mathrm{Z}}\left[R_{\mathrm{Z}, \mathrm{AA}}=1.07 \mathrm{~F}_{10.7, \mathrm{AA}}-63.8\right]$, this corresponds to $R_{\mathrm{Z}, \mathrm{AA}}=130$, above the amplitude of an average cycle $\left[R_{\mathrm{Z} \text {, ave }}=115 \pm 40\right.$ (Pesnell, 2008b) $]$ but below the predicted amplitude of Hathaway and Wilson (2006). This is the first prediction listed in Table 1. The change from $R_{\mathrm{Z}}$ to $\mathrm{F}_{10.7}$ and from aa to Ap has reduced the predicted amplitude by 30 sunspot numbers. By encompassing over 1200 days in the average, it is impossible to look for precursors at later times. We now examine the behavior of the precursor with the width of the Gaussian smoothing filter.

\section{Variation with the Width of the Gaussian Filter}

One complication of the geomagnetic-precursor pair forecast method is determining the location in time of the precursor peak. One reason why the precursor peak occurred in late 2003 and not closer to solar minimum could be the width of the Gaussian filter used in the analysis and the extraordinary level of solar activity in late 2003. This width also determines the temporal cutoff of the data. A filter with width $\sigma_{\mathrm{WG}}$ cannot be used to study effects nearer than about $2 \sigma_{\mathrm{WG}}$ from the end of the time series.

Versions of the Ap- $\mathrm{F}_{10.7}$ precursor function using different widths of the Gaussian filter are shown in Figure 6 . The widths were set to $\sigma_{\mathrm{WG}}=81,150$, and 310 days, with a cutoff at 


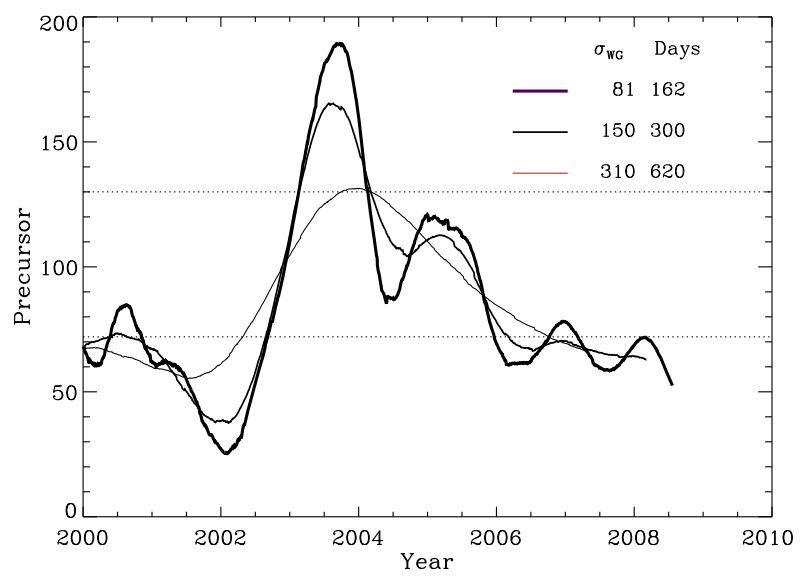

Figure 6 Versions of the Ap-F107 precursor function using different widths of the Gaussian filter. The widths were set to $\sigma_{\mathrm{WG}}=81,150$, and 310 days, with a cutoff at $2 \sigma_{\mathrm{WG}}$ before the end of the time series (the days column.) As $\sigma_{\mathrm{WG}}$ decreases, the value at the peak in 2003 increases, while a second peak in 2005, invisible in the $\sigma_{\mathrm{WG}}=310$ curve appears and becomes better resolved as $\sigma_{\mathrm{WG}}$ decreases to 150 . The shortest filter shows four distinct peaks from 2003 to 2008. The horizontal dotted lines show the prediction for the $\sigma_{\mathrm{WG}}=310$ - and 81-day precursors, the former at its peak and the latter at the peak coinciding with recurrent activity.

Figure 7 The variation of the height and date of the precursor peak in mid-2003 to early 2004. The Ap- $\mathrm{F}_{10.7}$ precursor function was evaluated with different widths of the Gaussian filter. As $\sigma_{\mathrm{WG}}$ increases, the value at the peak in 2003 decreases, while the date of the peak moves toward later times.

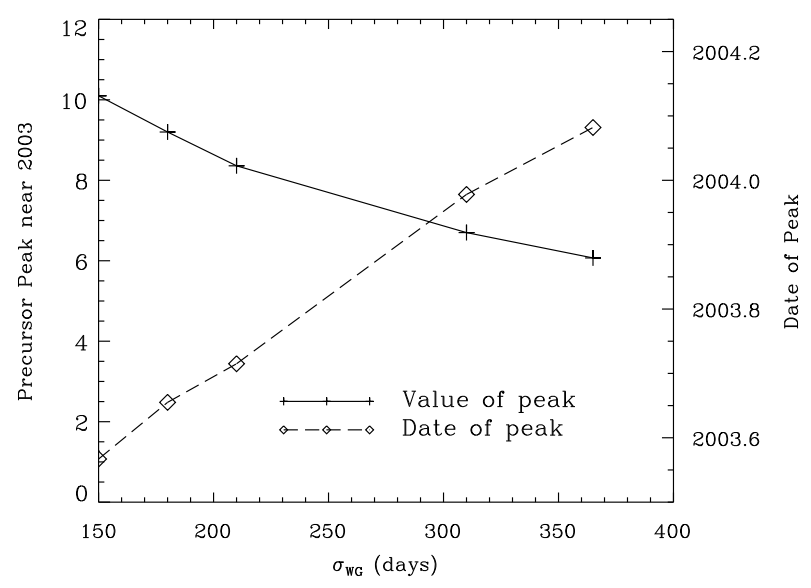

$\pm 2 \sigma_{\mathrm{WG}}$ (the days column). For each filter width the predictor function in Equation (2) was evaluated for all values of the precursor. This means that the predicted amplitude of Solar Cycle 24 can be read off the plot, leaving the choosing of the correct point in time as the remaining issue.

Four peaks are present between 2003 and 2008 in the precursor using the narrowest filter length $\left[\sigma_{\mathrm{WG}}=81\right.$ day]. As shown in Figure 7, the location of the peak in 2003 and its value changes in a systematic way with the filter width used in the analysis. The value of the peak in 2003 increases by $40 \%$ as the filter width decreases by almost $30 \%$ (Figure 7). The timing of the peak also shifts, moving back in time as the filter width is decreased. This correlated behavior of the peak and timing is due to the filter broadening to include two peaks of activity, one in 2003 and the other in 2005 . The peak in 2005 can be seen in the 
$\sigma_{\mathrm{WG}}=150$ days, but decreases to a shoulder in the $\sigma_{\mathrm{WG}}=210$ curve, and becomes invisible when $\sigma_{\mathrm{WG}}$ is increased to 310 .

Comparing the sunspot number predicted using the $\sigma_{\mathrm{WG}}=150$ and 310 day precursor values in 2005 ( $\mathrm{Ap}_{\mathrm{p}}=4.8$ and 4.0, respectively) in the correlation functions of Equation (2) gives estimated amplitudes of $R_{\mathrm{Z} \text {,aa }}=115$ and 105, the amplitude of an average cycle. This variation implies that an uncertainty of at least ten sunspot numbers should be assigned to the prediction on this basis alone.

Whether the Russell-McPherron effect is causing a modulation of the precursor cannot be addressed by this analysis. The width of the $\sigma_{\mathrm{WG}}=310$ day filter (FWHM $=730$ days) is wider than the seasonal dependence. When the filter length is reduced to 81 days, the seasonal dependence becomes more visible, but a lack of strict periodicity in this effect means that it is not averaged out by the filtering and must still be understood. The estimated uncertainty caused by this effect is eight in the predicted values of $\mathrm{F}_{10.7}$ and $R_{\mathrm{Z}}$.

Combining the formal error in Equation $(2)( \pm 10)$, the uncertainty in the value of the precursor $( \pm 10)$, and the variation from the Russell-McPherron effect $[ \pm 8]$ in a root-meansquare gives an error estimate of \pm 16 , which we rounded to a prediction uncertainty of \pm 20 . The uncertainty in the timing of the precursor peak is about 0.5 years based on the width of the peaks in the narrowest filters (Figure 6).

This analysis has not resolved which is the appropriate peak to use as a precursor of Cycle 24. The timing of the precursor could be related to the level of recurrent geomagnetic activity, which we now examine using a recurrence index.

\section{Comparison with a Recurrence Index}

The accuracy of the geomagnetic precursor is ascribed to its being sensitive to recurrent activity on the Sun. Geomagnetic activity near solar maximum is primarily driven by solar activity related to sunspots and active regions. These drivers are randomly distributed in longitude and relatively short lived, so the geomagnetic activity between successive solar rotations is uncorrelated. Even long-lived active regions will produce flares and CMEs at random times as they traverse the visible disk of the Sun. By comparison, the long-lived coronal holes present on the Sun during the decline from solar maximum are relatively fixed in heliographic longitude and emit high-speed streams that can strike the Earth every 27 days, driving periodic geomagnetic activity that can be used as a proxy for recurrent activity on the Sun. This can be represented with a recurrent index [RI] using an Ap that is similar to the one defined by Sargent (1985) using aa as the geomagnetic indicator.

We define RI as the correlation in Ap from one Bartels rotation to the next:

$$
\mathrm{RI}_{\mathrm{i}}=\sum_{j=1}^{27} \frac{\left(\mathrm{Ap}_{i, j}-\langle\mathrm{Ap}\rangle_{i}\right)\left(\mathrm{Ap}_{i-1, j}-\langle\mathrm{Ap}\rangle_{i-1}\right)}{\sigma_{i} \sigma_{i-1}},
$$

where $\langle\mathrm{Ap}\rangle_{i}$ is the average of Ap over Bartels rotation $i, \sigma_{i}$ is the standard deviation from the average in that interval, and the time assigned is the beginning of rotation $i$, the middle of the combined intervals. (A Bartels rotation number is the number of 27-day solar-rotation periods since the first began on 8 February 1932.) RI and the Ap- $F_{10.7}$ precursor from 1950 through 2008 are plotted in Figure 8. The level of recurrent activity is highest near solar minimum and lowest near solar maximum.

Each of the precursor peaks prior to Solar Cycles 20-23 coincides with a local maximum in RI exceeding 0.3 . If recurrent activity were the source of the precursor peak in 


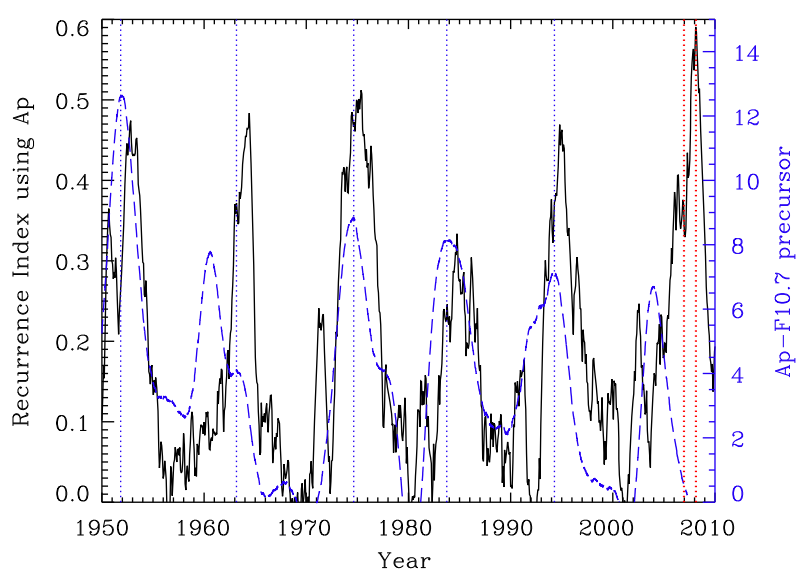

Figure 8 The recurrence index [RI] defined by the correlation of Ap between one Bartel rotation and the next (Equation (3)). The curve was smoothed over 15 rotations. Shown as a blue dashed line is the Ap- $\mathrm{F}_{10.7}$ precursor function from Figure 4. Vertical blue dashed lines show the maxima of the precursor used to construct the correlation function in Figure 5. The vertical red dotted lines show the minima in 2006 and 2008. High values of RI (approaching and exceeding 0.5) are seen during solar minimum, while RI is close to (and sometimes can dip below) zero at solar maximum. Solar activity was dominated by several large active regions in the second half of 2003, which reduced RI to near zero. As we neared solar minimum in 2008, RI reached values higher than previously seen, but has since declined. A recurrence index derived from aa shows similar behavior.

2003, a peak in RI should coincide with that precursor peak. The temporal variation of RI since 2000 and the Ap- $\mathrm{F}_{10.7}$ geomagnetic-precursor pairs for different smoothing widths are plotted in Figure 9. A local minimum in the recurrent index is indeed present in late 2003, with RI approaching zero. If the filter width is increased to $\sigma_{\mathrm{WG}}=365$ days, the peak moves into early 2004, where RI is increasing but remains lower than 0.2. Even the second possible precursor peak in 2005 (calculated with the narrower filters) is not coincident with a local maximum of recurrent activity. It is not until 2008 that unambiguous recurrent geomagnetic activity is present, and it is at this time that a fourth peak appears in the narrowest filters. This precursor is $\mathrm{Ap}_{\mathrm{p}}=0.1$ and gives $\mathrm{F}_{10.7,24}=122$ (Table 1$)$.

\section{Conclusions}

Using Ap and $\mathrm{F}_{10.7}$ as a geomagnetic-precursor pair gives a predicted amplitude for Solar Cycle 24 that is smaller than a similar analysis using aa and $R_{\mathrm{Z}}$. Once the interplanetary component of Ap is derived and used as a precursor, we obtained an $\mathrm{F}_{10.7}$ amplitude of $122 \pm 20$ and a sunspot number of $65 \pm 20$ for Solar Cycle 24, with the peak of solar activity in May 2014. The combination of $R_{\mathrm{Z}}$ underestimating $\mathrm{F}_{10.7}$ and aa overestimating $\mathrm{Ap}$ in the latter parts of Solar Cycle 23 leads to a prediction of a lower-amplitude cycle when Ap and $\mathrm{F}_{10.7}$ are used as the precursor pair. Requiring significant recurrent activity to be present also delays the timing of the peak and allows the precursor to drop well below the 2003 value. This prediction for a below-average Solar Cycle 24 agrees with the SOlar Dynamic Amplitude (SODA) Index prediction of Schatten (2005) and others that use the solar polar magnetic field as a precursor of future solar activity (Svalgaard, Cliver, and Kamide, 2005).

We have examined how the precursor's value and location varies with the width of the smoothing filter and how the presence of multiple peaks in the precursor can lead to misiden- 


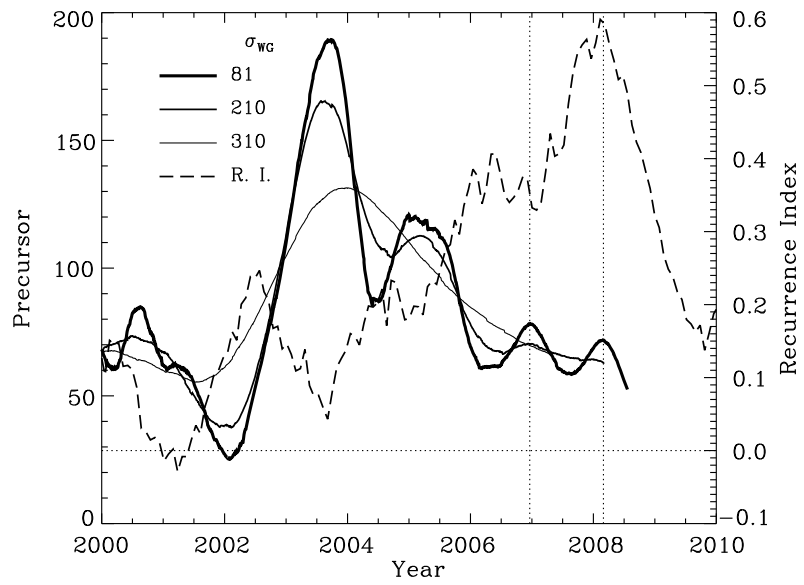

Figure 9 Versions of the $\mathrm{Ap}-\mathrm{F}_{10.7}$ geomagnetic precursor using widths of $\sigma_{\mathrm{WG}}=81,150$, and 310 days. Plotted as a dashed line is the recurrence index defined by Equation (3) smoothed over 15 rotations. The horizontal dotted line shows the zero level of RI. If recurrent activity were the source of the precursor peak in 2003, a peak in the recurrent index should coincide with the precursor peak in 2003. A local minimum in the recurrent index is indeed present at that time, with the index approaching zero. Smoothing of the recurrent index over more rotations does not remove the dip in 2003. Even the second possible precursor peak in 2005 is not coincident with a local maximum of recurrent activity. The widest peaks represent averages over episodes of non-recurrent and recurrent activity. That tends to increase the precursor by emphasizing the extremely high levels of activity in late 2003 and smearing the later, narrower peaks.

tifying the timing of the precursor peak. This ambiguity of multiple precursor peaks can be resolved by requiring that substantial recurrent activity coincides with the peak. This allows narrower filters to be applied during the smoothing step, reducing edge effects and revealing weaker features.

Brown (1992) showed that predictions of the amplitude of Solar Cycle 22 using bivariate functions (such as the aa $-R_{\mathrm{Z}}$ and $\mathrm{Ap}-\mathrm{F}_{10.7}$ precursor pairs discussed here) were less accurate than those using only the geomagnetic index at solar minimum as the predictor function. This may be repeated in the amplitude predictions of Solar Cycle 24. However, by delaying the evaluation of the precursor function until almost solar minimum, the Ap- $\mathrm{F}_{10.7}$ precursor is reduced to the minimum-Ap precursor.

From a comparison with an index of recurrent geomagnetic activity, we showed that the peak of the precursor function in 2003 is associated with non-recurrent geomagnetic activity. In previous cycles the precursor peak coincided with a peak in the recurrence index defined in Equation (3). Two more precursor peaks, accompanied by some recurrent activity, were found in 2005 and 2006 using narrower filter widths. The behavior of the Solar Cycle 24 precursor would then be similar to the one preceding Solar Cycle 20, another small cycle. That is not true of the precursor peak in 2003, which is dominated by activity with a very low value of RI. Our narrowest filter ( $\sigma_{\mathrm{WG}}=81$ days) has a peak in early 2008 with a value of approximately 0.1 that coincides with $\mathrm{RI} \approx 0.6$. When used in Equation $(2)$, this corresponds to $R_{24, \mathrm{AA}}=65$.

Another precursor prediction uses the strength of the polar magnetic fields to estimate the amplitude of an upcoming solar cycle. Using this technique, Svalgaard, Cliver, and Kamide (2005) and Schatten (2005) predicted small amplitudes (75 \pm 8 and $80 \pm 20$, respectively) based on the weak polar fields observed on the Sun during the decline of Cycle 23. This precursor is based on solar-dynamo theory, where the polar fields at solar minimum form 
the seed for the next cycle's toroidal fields and activity level. Predictions for Cycle 23 using the polar-field precursor also tended to be lower and in better agreement with the actual amplitude of Cycle 23 than the geomagnetic precursor.

The earliest SODA Index prediction for Solar Cycle 24 was reported by Schatten (2005). SODA is designed to be a continuously updated prediction (Schatten and Pesnell, 1993). Looking at the SODA Index over the years between the first prediction and solar minimum gives an average prediction of $138 \pm 2.5$ for $\mathrm{F}_{10.7}$ or $84 \pm 2.5$ for $R_{\mathrm{Z}}$. This consistency in the prediction is in contrast to the change in the geomagnetic precursor prediction of 50 sunspot numbers $(40 \%)$ during the same period of time. By relying on a particular time in the decline of the solar cycle, geomagnetic-precursor pairs tend to be a upper limit until much closer to solar minimum than is the polar field precursor.

Solar polar magnetic fields are a direct measurement of one aspect of the solar dynamo, and it is reasonable to expect a precursor relationship between those fields and future solar activity. In addition, the solar polar field strength was slowly varying near a peak value in the recent solar minimum, lending credence to a prediction using that field strength. Geomagnetic precursors are at least one layer of abstraction removed from the solar magnetic fields that presage the next cycle. Regardless of which solar magnetic fields are the source of the geomagnetic activity, the uncertainty of that coupling layer may be increasing. An understanding of this layer, which could drift in time, is needed to link the response of the Earth to the future behavior of the Sun. Geomagnetic activity also tends to decrease to very low levels at solar minimum, adding to the difficulty of using aa and Ap as a precursor.

Whether $\mathrm{F}_{10.7}$ or $R_{\mathrm{Z}}$ more accurately represents solar activity for use in geomagnetic precursors can only be assessed by knowing that they currently differ and monitoring their future behavior. This research has shown that having solar-activity indices that are accurate and cover long times is an important part of solar-cycle prediction. As predictions of the next solar cycle are made at ever-earlier times before solar minimum, the sensitivity of geomagnetic precursors to filter length is quite troubling. A requirement of coincident recurrent activity may ensure that a proper prediction is made.

Acknowledgements This work was supported by the Solar Dynamics Observatory at NASA Goddard Space Flight Center. Daily values of $R_{\mathrm{Z}}$ and Adjusted $\mathrm{F}_{10.7}$ (normalized to $1 \mathrm{AU}$ ) were obtained from the National Geophysical Data Center in Boulder, CO, USA. Values of Ap were obtained from the monthly files at ftp.ngdc.noaa.gov/STP/GEOMAGNETIC_DATA/INDICES/KP_AP/. Values of aa through 2007 were obtained from ftp.ngdc.noaa.gov/STP/GEOMAGNETIC_DATA/AASTAR/aaindex. More recent values of aa were obtained from isgi.cetp.ipsl.fr/des_aa.htm.

Open Access This article is distributed under the terms of the Creative Commons Attribution License which permits any use, distribution, and reproduction in any medium, provided the original author(s) and the source are credited.

\section{Appendix: Sources and Preparation of Data}

Predicting solar activity with a geomagnetic-precursor pair requires an index of geomagnetic activity and an index of solar activity. The solar-activity indices and two geomagnetic indices used in this article are described here.

$R_{\mathrm{Z}}$

The sunspot number $\left[R_{\mathrm{Z}}\right]$ is often used as a long-term index of solar activity. It has been measured or derived for roughly 400 years and is the source of much of our knowledge of 
the evolution of solar activity. Several versions of the sunspot-number record, such as the International, American, and group, are available; we used the International. Problems in the homogeneity of the $R_{\mathrm{Z}}$ time series are described by Cliver and Svalgaard (2007).

$\mathrm{F}_{10.7}$

The solar spectral irradiance at a wavelength of $10.7 \mathrm{~cm}\left[\mathrm{~F}_{10.7}\right]$ is a well-known index of solar activity, which has been measured on a daily basis since 1947 (Covington, 1969; Tapping and Charrois, 1994). $\mathrm{F}_{10.7}$ peaks at solar maximum and fades to around $60 \times$ $10^{-22} \mathrm{~W} \mathrm{~m}^{-2} \mathrm{~Hz}^{-1} \equiv 60 \mathrm{SFU}$ at solar minimum. Because it has a latency of 24 hours, $\mathrm{F}_{10.7}$ is often used as a proxy for solar activity in models of the thermosphere (to understand satellite drag) and ionosphere (for radio-wave interference). Therefore, predictions of $\mathrm{F}_{10.7}$ are very useful to operators of satellites and the space-weather community.

aa

The aa index is the average of magnetic activity measured at two antipodal observatories at an invariant magnetic latitude $[\Lambda]$ of about $\pm 50^{\circ}$ but located at diametrically opposite sites on the Earth (Mayaud, 1980). The antipodal average is used to approximately cancel the diurnal and annual variations in the aa index (Mayaud, 1972).

Ap

Another widely used geomagnetic index is the daily equivalent planetary amplitude or Ap index. It is constructed using amplitudes from 13 stations to approximately account for the local time and annual variations. The aa and Ap indices have a similar temporal dependence because they are both based on the three-hourly $\mathrm{K}$ indices measured at the stations in the same network of geomagnetic observatories (Menvielle and Berthelier, 1991), but Ap is derived from a larger network of stations.

\section{Active Region Data}

The number of active regions in both hemispheres of the Sun were derived from the active regions reported for each year since 1965 by the National Geophysical Data Center (NGDC) website. Before 1965 the data values were obtained from the NGDC as yearly files containing the measured active-region locations and areas from the Greenwich Observatory (ftp.ngdc.noaa.gov/STP/SOLAR_DATA/SUNSPOT_REGIONS/Greenwich/). The overlap years were averaged. A summary file of annual active-region areas in the northern and southern hemispheres (ftp.ngdc.noaa.gov/STP/SOLAR_DATA/SUNSPOT_REGIONS/ Greenwich/docs/ss_area.n_s) showed similar behavior.

\section{References}

Biesecker, D., the Solar Cycle 24 Prediction Panel: 2007, Consensus statement of the solar cycle 24 prediction panel, released March 2007. www.swpc.noaa.gov/SolarCycle/SC24/.

Brown, G.M.: 1992, The peak of solar cycle 22 - Predictions in retrospect. Ann. Geophys. 10, 453.

Cliver, E.W., Svalgaard, L.: 2007, Origins of the Wolf sunspot number series: Geomagnetic underpinning. AGU Fall 2007 Meeting Abs., Abstract A1109.

Covington, A.E.: 1969, Solar radio emission at $10.7 \mathrm{~cm}, 1947-1968$. J. Roy. Astron. Soc. Can. 63, 125. 
Crooker, N.U., Cliver, E.W., Tsurutani, B.T.: 1992, The semiannual variation of great geomagnetic storms and the postshock Russell-McPherron effect preceding coronal mass ejecta. Geophys. Res. Lett. 19 , 429. DOI.

Feynman, J.: 1982, Geomagnetic and solar wind cycles, 1900-1975. J. Geophys. Res. 87, 6153.

Hathaway, D.H., Wilson, R.M.: 2006, Geomagnetic activity indicates large amplitude for sunspot cycle 24. Geophys. Res. Lett. 33, L18101. DOI.

Hathaway, D.H., Wilson, R.M., Reichman, E.J.: 1994, The shape of the sunspot cycle. Solar Phys. $151,177$. DOI.

Hathaway, D.H., Wilson, R.M., Reichman, E.J.: 1999, A synthesis of solar cycle prediction techniques. J. Geophys. Res. 104, 375.

Joselyn, J.A., Anderson, J.B., Coffey, H., Harvey, K., Hathaway, D., Heckman, G., Hildner, E., Mende, W., Schatten, K., Thompson, R., Thomson, A.W.P., White, O.R.: 1997, Panel achieves consensus prediction of solar cycle 23. Eos Trans. AGU 78, 205.

Kryachko, A.V., Nusinov, A.A.: 2008, Standard prediction of solar cycles. Geomagn. Aeron. 48, 145. DOI.

Li, K.J., Gao, P.X., Zhan, L.S.: 2009, The long-term behavior of the North-South asymmetry of sunspot activity. Solar Phys. 254, 145. DOI.

Livingston, W., Penn, M.J., Svalgaard, L.: 2012, Decreasing sunspot magnetic fields explain unique $10.7 \mathrm{~cm}$ radio flux. Astrophys. J. Lett. 757, L8. DOI.

Luhmann, J.G., Li, Y., Arge, C.N., Gazis, P.R., Ulrich, R.: 2002, Solar cycle changes in coronal holes and space weather cycles. J. Geophys. Res. 107, 1154. DOI.

Mayaud, P.-N.: 1972, The aa indices: A 100-year series characterizing the magnetic activity. J. Geophys. Res. 77, 6870. DOI.

Mayaud, P.-N.: 1980, Derivation, Meaning, and Use of Geomagnetic Indices, Geophys. Monogr. 22, AGU, Washington, 82.

McIntosh, S.W., Leamon, R.J., Gurman, J.B., Olive, J.-P., Cirtain, J.W., Hathaway, D.H., Burkepile, J., Miesch, M., Markel, R.S., Sitongia, L.: 2013, Hemispheric asymmetries of solar photospheric magnetism: Radiative, particulate, and heliospheric impacts. Astrophys. J. 765, 146. DOI.

Menvielle, M., Berthelier, A.: 1991, The K-derived planetary indices: Description and availability. Rev. Geophys. 29, 415.

Ohl, A.I.: 1966, Wolf's number prediction for the maximum of the cycle 20. Soln. Dannye 12, 84.

Ohl, A.I., Ohl, G.I.: 1979, A new method of very long-term prediction of solar activity. In: Donnelly, R. (ed.) Solar-Terrestrial Predictions Proc. 2, 258. NOAA/Space Environment Laboratory.

Pesnell, W.D.: 2008a, Predicting solar activity: Today, tomorrow, next year. In: 88th Ann. Meeting Am. Met. Soc., 107.

Pesnell, W.D.: 2008b, Predictions of solar cycle 24. Solar Phys. 252, 209. DOI.

Pesnell, W.D.: 2012, Solar cycle predictions (Invited review). Solar Phys. 281, 507. DOI.

Pevtsov, A.A., Bertello, L., Tlatov, A.G., Kilcik, A., Nagovitsyn, Y.A., Cliver, E.W.: 2013, Cyclic and longterm variation of sunspot magnetic fields. Solar Phys. DOI.

Russell, C.T., McPherron, R.L.: 1973, Semiannual variation of geomagnetic activity. J. Geophys. Res. 78, 92.

Sargent, H.H. III: 1978, A prediction for the next sunspot cycle. In: 28th IEEE Vehicular Technology Conference, 1978 28, 490. DOI.

Sargent, H.H. III: 1985, Recurrent geomagnetic activity - Evidence for long-lived stability in solar wind structure. J. Geophys. Res. 90, 1425.

Schatten, K.: 2005, Fair space weather for solar cycle 24. Geophys. Res. Lett. 32, L21106. DOI.

Schatten, K.H., Pesnell, W.D.: 1993, An early solar dynamo prediction: Cycle 23 is approximately cycle 22. Geophys. Res. Lett. 20, 2275. DOI.

Svalgaard, L.: 2009, The solar radio microwave flux. Accessed on December 19, 2009. www.leif.org/research/SolarRadioFlux.pdf.

Svalgaard, L.: 2012, How well do we know the sunspot number? In: Mandrini, C.H., Webb, D.F. (eds.) IAU Symp. 286, Cambridge Univ. Press, Cambridge, 27. DOI.

Svalgaard, L., Cliver, E.W., Le Sager, P.: 2004, IHV: A new long-term geomagnetic index. Adv. Space Res. 34, 436. DOI.

Svalgaard, L., Cliver, E.W., Kamide, Y.: 2005, Cycle 24: The smallest sunspot cycle in 100 years? Geophys. Res. Lett. 32. DOI.

Tapping, K.F., Charrois, D.P.: 1994, Limits to the accuracy of the $10.7 \mathrm{~cm}$ flux. Solar Phys. 150, 305. DOI.

Tapping, K.F., Valdés, J.J.: 2011, Did the Sun change its behaviour during the decline of cycle 23 and into cycle 24? Solar Phys. 272, 337. DOI.

Temmer, M., Veronig, A., Hanslmeier, A., Otruba, W., Messerotti, M.: 2001, Statistical analysis of solar H $\alpha$ flares. Astron. Astrophys. 375, 1049. DOI.

Wang, Y.-M., Sheeley, N.R.: 2009, Understanding the geomagnetic precursor of the solar cycle. Astrophys. J. 694, 11. DOI. 